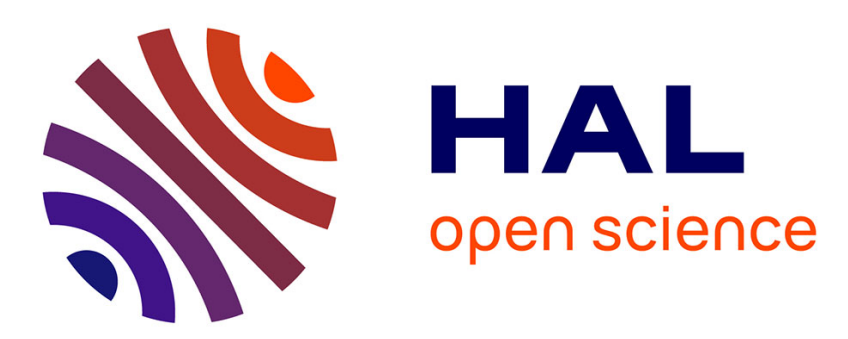

\title{
Oscillations Conditions in Homogenous Systems
}

Denis Efimov, Wilfrid Perruquetti

\section{To cite this version:}

Denis Efimov, Wilfrid Perruquetti. Oscillations Conditions in Homogenous Systems. 8th IFAC Symposium on Nonlinear Control Systems, Sep 2010, Bologna, Italy. pp.1379-1384. hal-00561003

\section{HAL Id: hal-00561003 \\ https://hal.science/hal-00561003}

Submitted on 14 Oct 2011

HAL is a multi-disciplinary open access archive for the deposit and dissemination of scientific research documents, whether they are published or not. The documents may come from teaching and research institutions in France or abroad, or from public or private research centers.
L'archive ouverte pluridisciplinaire HAL, est destinée au dépôt et à la diffusion de documents scientifiques de niveau recherche, publiés ou non, émanant des établissements d'enseignement et de recherche français ou étrangers, des laboratoires publics ou privés. 


\title{
Oscillations Conditions in Homogenous Systems
}

\author{
Efimov D. ${ }^{1}$, Perruquetti W. ${ }^{2,3}$ \\ ${ }^{1}$ University of Bordeaux, IMS-lab, Automatic control group \\ 351 cours de la libération, 33405 Talence, France \\ denis.efimov@,ims-bordeaux.fr \\ ${ }^{2}$ LAGIS (UMR-CNRS 8146), Ecole Centrale de Lille, BP 48, Cité Scientifique, 59651 \\ Villeneuve-d'Ascq, France \\ ${ }^{3}$ ALIEN project at INRIA - LNE, Parc Scientifique de la Haute Borne 40, \\ avenue Halley Bât.A, Park Plaza 59650 Villeneuve d'Ascq, France \\ wilfrid.perruquetti@,inria.fr
}

\begin{abstract}
The problem of oscillations detection for nonlinear systems is addressed. The notion of homogeneity in bi-limit from (Andrieu, et al., 2008) is extended to local homogeneity. Next, sufficient conditions of oscillations existence for locally homogeneous systems are formulated. The proposed approach allows one to estimate the number of limit cycles and the regions of their location. Efficiency of the technique is demonstrated on two academic examples.
\end{abstract}

\section{INTRODUCTION}

The homogeneity is a propriety of nonlinear dynamical systems introduced more than thirty years ago (Rothschild and Stein, 1976) meaning that the state vector rescaling does not change the system behavior. Thus, the behavior of the system trajectories on a suitably defined sphere around the origin can be extended to the whole state space. This property is used for stability analysis (Andrieu, et al., 2008; Bacciotti and Rosier, 2001; Hermes, 1991a; 1991b; Rosier, 1992), systems approximation (Hermes, 1991a), stabilization (Bhat and Bernstein, 2005; Grüne, 2000; Kawski, 1991; Moulay and Perruquetti, 2006; Sepulchre and Aeyels, 1996), and observation (Andrieu, et al., 2008). In the work (Andrieu, et al., 2008) the homogeneity in the bi-limit is introduced, that is homogeneity with different weights and approximating functions at a vicinity of the origin and far outside.

Despite all these works are devoted to studying stability-like behavior of the origin during recent years an interest to more complex dynamical systems behavior including oscillatory (periodical or chaotic) has grown significantly (Fradkov and Pogromsky, 1998; Leonov, et al., 1995; Martinez, et al., 2003; Di Meglio, et al., 2009; Sinègre, et al., 2005). An important and useful concept for studying irregular oscillations is the theory introduced by V.A. Yakubovich (Yakubovich, 1973; 1975; Yakubovich and Tomberg, 1989). Recently proposed conditions of oscillations in the sense of Yakubovich (Efimov and Fradkov, 2009) are based on two Lyapunov functions existence. The first Lyapunov function ensures local instability of the origin, while the second provides global boundedness of the system trajectories, that under some mild conditions implies existence of oscillations.

Such existence of two Lyapunov functions nicely interacts with homogeneity in the bi-limit: in both cases two subspaces of the system operation are considered separately. The pecu- liarity of oscillating systems is that the instability around the origin is required. In the present work we are going to develop the results from (Andrieu, et al., 2008; Efimov and Fradkov, 2009) proposing conditions of oscillations for homogeneous systems, relaxing the (Efimov and Fradkov, 2009) conditions conservatism and extending them to the case of several limit cycles existence, when the system may be asymptotically stable around the origin and at infinity with instability regions among them. An extension of the instability Lyapunov theorems for homogeneous systems is also presented.

The outline of the paper is as follows. The homogeneity and the oscillatory properties are introduced in Section 2. Main results are presented in Section 3. Some applications of the proposed results are discussed in Section 4.

\section{PRELIMINARIES}

Consider the nonlinear dynamical system:

$$
\dot{\mathbf{x}}=\mathbf{f}(\mathbf{x}) \text {, }
$$

where $\mathbf{x} \in R^{n}$ is the state vector, $\mathbf{f}: R^{n} \rightarrow R^{n}, \mathbf{f}(0)=0$ is a nonlinear function ensuring existence and uniqueness of the system (1) solutions (for any initial conditions $\mathbf{x}_{0} \in R^{n}$ the solution $\mathbf{x}\left(t, \mathbf{x}_{0}\right)$ of the system (1) is defined at least locally for $t \leq T$, further we will simply write $\mathbf{x}(t)$ if origin of initial conditions is clear from the context). If for all initial conditions $\mathbf{x}_{0} \in R^{n}$ the solutions are defined for all $t \geq 0$ then the system (1) is called forward complete.

The system (1) is called locally or globally asymptotically stable (at the origin) if the standard conditions are satisfied (Khalil, 2002). The asymptotic stability of the system (1) with respect to an invariant set is treated in the sense of (Lin, et al., 1996). 
Recall that a continuous function $\alpha: R_{+} \rightarrow R_{+}$belongs to the class $K$ if $\alpha(0)=0$ and the function is strictly increasing. The function $\alpha: R_{+} \rightarrow R_{+}$belongs to the class $K_{\infty}$ if $\alpha \in K$ and it is increasing to infinity.

\subsection{Homogeneity}

For any $r_{i}>0, i=\overline{1, n}$ and $\lambda \geq 0$ define the dilation matrix $\boldsymbol{\Lambda}_{\mathbf{r}}=\operatorname{diag}\left\{\lambda^{r_{i}}\right\}_{i=1}^{n}$ and the vector of weights $\mathbf{r}=\left[r_{1} \ldots r_{n}\right]^{T}$. For any $r_{i}>0, i=\overline{1, n}$ the homogeneous norm can be defined as follows

$$
|\mathbf{x}|_{\mathbf{r}}=\sum_{i=1}^{n}\left|x_{i}\right|^{1 / r_{i}}
$$

There exist two functions $\underline{\sigma}_{\mathbf{r}}, \bar{\sigma}_{\mathbf{r}} \in K_{\infty}$ such that

$$
\underline{\sigma}_{\mathbf{r}}\left(|\mathbf{x}|_{\mathbf{r}}\right) \leq|\mathbf{x}| \leq \bar{\sigma}_{\mathbf{r}}\left(|\mathbf{x}|_{\mathbf{r}}\right)
$$

for all $\mathbf{x} \in R^{n}$ (the functions $\underline{\sigma}_{\mathbf{r}}, \bar{\sigma}_{\mathbf{r}}$ define Euclidean norm deviations with respect to the homogeneous norm, i.e. if $\mathbf{x} \in R^{n} \quad$ with the property $\quad|\mathbf{x}|_{\mathbf{r}}=\xi$, then $\left.\underline{\sigma}_{\mathbf{r}}(\xi) \leq|\mathbf{x}| \leq \bar{\sigma}_{\mathbf{r}}(\xi)\right)$. The homogeneous norm has an important property that $\left|\boldsymbol{\Lambda}_{\mathbf{r}} \mathbf{x}\right|_{\mathbf{r}}=\lambda|\mathbf{x}|_{\mathbf{r}}$. Define $S_{\mathbf{r}}=\left\{\mathbf{x} \in R^{n}:|\mathbf{x}|_{\mathbf{r}}=1\right\}$.

Definition 1 . The function $g: R^{n} \rightarrow R$ is called $\mathbf{r}$ homogeneous $\left(r_{i}>0, i=\overline{1, n}\right.$ ) iffor any $\mathbf{x} \in R^{n}$

$$
g\left(\boldsymbol{\Lambda}_{\mathbf{r}} \mathbf{x}\right)=\lambda^{d} g(\mathbf{x})
$$

for some $d \geq 0$ and all $\lambda \geq 0$.

The system (1) is called $\mathbf{r}$-homogeneous $\left(r_{i}>0, i=\overline{1, n}\right)$ if for any $\mathbf{x} \in R^{n}$

$$
\mathbf{f}\left(\boldsymbol{\Lambda}_{\mathbf{r}} \mathbf{x}\right)=\lambda^{d} \boldsymbol{\Lambda}_{\mathbf{r}} \mathbf{f}(\mathbf{x})
$$

for some $d \geq-\min _{1 \leq i \leq n} r_{i}$ and all $\lambda \geq 0$.

The notation $D V(\mathbf{x}) \mathbf{f}(\mathbf{x})$ stands for directional derivative of the differentiable function $V$ with respect to the vector field $\mathbf{f}$, and for Dini derivative in the direction of $\mathbf{f}$

$$
D V(\mathbf{x}) \mathbf{f}(\mathbf{x})=\lim _{t \rightarrow 0^{+}} \inf \frac{V(\mathbf{x}+t \mathbf{f}(\mathbf{x}))-V(\mathbf{x})}{t}
$$

if the function $V$ is Lipschitz continuous.

The or em 1 (Rosier, 1992). For the system (1) with $\mathbf{r}$ homogeneous and continuous function $\mathbf{f}: R^{n} \rightarrow R^{n}$ the following properties are equivalent:

- the system (1) is (locally) asymptotically stable;

- there exists continuously differentiable homogeneous

Lyapunov function $V: R^{n} \rightarrow R_{+}$such that for all $\mathbf{x} \in R^{n}$,

$$
\begin{gathered}
\alpha_{1}(|\mathbf{x}|) \leq V(\mathbf{x}) \leq \alpha_{2}(|\mathbf{x}|), D V(\mathbf{x}) \mathbf{f}(\mathbf{x}) \leq-\alpha(|\mathbf{x}|), \\
V\left(\boldsymbol{\Lambda}_{\mathbf{r}} \mathbf{x}\right)=\lambda^{k} V(\mathbf{x}), k \geq 0,
\end{gathered}
$$

for some $\alpha_{1}, \alpha_{2} \in K_{\infty}, \alpha \in K$.

Note, that the continuity of the function $\mathbf{f}$ is required for the necessary part only. The $\mathbf{r}$-homogeneity property used in
Definition 1 and Theorem 1 is introduced for some $\mathbf{r}>0$ and all $\lambda \geq 0$. Restricting the set of admissible values for $\lambda$ we can introduce local homogeneity (in the 0 -limit or in the $\infty$ limit (Andrieu, et al., 2008)).

Definition 2. The function $g: R^{n} \rightarrow R, g(0)=0$ is called $\quad\left(\mathbf{r}, \lambda_{0}, g_{0}\right)$-homogeneous $\quad\left(r_{i}>0, \quad i=\overline{1, n}\right.$; $g_{0}: R^{n} \rightarrow R, g_{0}(0)=0$ ) iffor any $\mathbf{x} \in S_{\mathbf{r}}$

$$
\lim _{\lambda \rightarrow \lambda_{0}} \lambda^{-d_{0}} g\left(\boldsymbol{\Lambda}_{\mathbf{r}} \mathbf{x}\right)-g_{0}(\mathbf{x})=0
$$

for some $d_{0} \geq 0$.

The system (1) is called $\left(\mathbf{r}, \lambda_{0}, \mathbf{f}_{0}\right)$-homogeneous $\left(r_{i}>0\right.$, $\left.i=\overline{1, n} ; \mathbf{f}_{0}: R^{n} \rightarrow R^{n}, \mathbf{f}_{0}(0)=0\right)$ if for any $\mathbf{x} \in S_{\mathbf{r}}$

$$
\lim _{\lambda \rightarrow \lambda_{0}} \lambda^{-d_{0}} \Lambda_{\mathbf{r}}^{-1} \mathbf{f}\left(\boldsymbol{\Lambda}_{\mathbf{r}} \mathbf{x}\right)-\mathbf{f}_{0}(\mathbf{x})=0
$$

for some $d_{0} \geq-\min _{1 \leq i \leq n} r_{i}$.

In the paper (Andrieu, et al., 2008) this definition has been introduced for $\lambda_{0}=0$ and $\lambda_{0}=+\infty$ (the function $g$ is called homogeneous in the bi-limit if it is simultaneously $\left(\mathbf{r}_{0}, 0, g_{0}\right)$-homogeneous and $\left(\mathbf{r}_{\infty},+\infty, g_{\infty}\right)$-homogeneous), the case $\lambda_{0}=0$ has been also treated in (Bacciotti and Rosier, 2001; Hermes, 1991b; Hong, 2002; Rosier, 1992). Note, that the system (1) can be also homogeneous in more than two limits (some examples are considered in Section 4).

If the pairs of functions $g, g_{0}$ and $\mathbf{f}, \mathbf{f}_{0}$ are continuous, then for any compact set $X \subset R^{n}$ and any $\varepsilon>0$ there exist $\underline{\lambda}_{\varepsilon} \leq \lambda_{0} \leq \bar{\lambda}_{\varepsilon}$ such that for all $\lambda \in\left(\underline{\lambda}_{\varepsilon}, \bar{\lambda}_{\varepsilon}\right)$ :

$$
\begin{gathered}
\sup _{\mathbf{x} \in S_{\mathbf{r}}}\left|\lambda^{-d_{0}} g\left(\boldsymbol{\Lambda}_{\mathbf{r}} \mathbf{x}\right)-g_{0}(\mathbf{x})\right| \leq \varepsilon, \\
\sup _{\mathbf{x} \in S_{\mathbf{r}}}\left|\lambda^{-d_{0}} \boldsymbol{\Lambda}_{\mathbf{r}}^{-1} \mathbf{f}\left(\boldsymbol{\Lambda}_{\mathbf{r}} \mathbf{x}\right)-\mathbf{f}_{0}(\mathbf{x})\right| \leq \varepsilon .
\end{gathered}
$$

The coefficients $r_{i}>0, i=\overline{1, n}$ are called weights, $d_{0}$ is the degree of homogeneity (it may depend on $\lambda_{0}$ ), $\mathbf{f}_{0}$ or $g_{0}$ are the approximating functions. Let us recall hereafter two useful results to be used later on.

Theorem 2 (Hermes, 1991a; Rosier, 1992). Let the system (1) be $\left(\mathbf{r}, 0, \mathbf{f}_{0}\right)$-homogeneous with the continuous functions $\mathbf{f}: R^{n} \rightarrow R^{n}$ and $\mathbf{f}_{0}: R^{n} \rightarrow R^{n}$. If the system $\dot{\mathbf{x}}=\mathbf{f}_{0}(\mathbf{x})$ is (locally) asymptotically stable, then the system (1) is also locally asymptotically stable.

Th e o r e m 3 (Andrieu, et al., 2008). Let the system (1) be $\left(\mathbf{r},+\infty, \mathbf{f}_{\infty}\right)$-homogeneous with the continuous functions $\mathbf{f}: R^{n} \rightarrow R^{n}$ and $\mathbf{f}_{\infty}: R^{n} \rightarrow R^{n}$. If the system $\dot{\mathbf{x}}=\mathbf{f}_{\infty}(\mathbf{x})$ is globally asymptotically stable, then there exists a compact invariant set $X_{\infty} \subset R^{n}$ such that the system (1) is globally asymptotically stable with respect to the set $X_{\infty}$.

The theorems 2 and 3 present results on the system (1) stabil- 
ity derived from the corresponding properties of the approximating systems for $\lambda_{0}=0$ or $\lambda_{0}=+\infty$. The converse Lyapunov theorem similar to Theorem 1 for the homogeneous in the bi-limit systems can be also found in (Andrieu, et al., 2008).

\subsection{Conditions of oscillations}

The function $g: R^{n} \rightarrow R$ is called monotone if the condition $x_{1} \leq x_{1}^{\prime}, \ldots, \quad x_{n} \leq x_{n}^{\prime} \quad$ implies that everywhere either $g\left(x_{1}, \ldots, x_{n}\right) \leq g\left(x_{1}^{\prime}, \ldots, x_{n}^{\prime}\right)$ or $g\left(x_{1}, \ldots, x_{n}\right) \geq g\left(x_{1}^{\prime}, \ldots, x_{n}^{\prime}\right)$.

Definition 3 (Yakubovich, 1973; Efimov and Fradkov, 2009). The solution $\mathbf{x}\left(t, \mathbf{x}_{0}\right)$ with $\mathbf{x}_{0} \in R^{n}$ of the system (1) is called $\left[\pi^{-}, \pi^{+}\right]$-oscillation with respect to the output $\psi=\eta(\mathbf{x})$ (where $\eta: R^{n} \rightarrow R$ is a continuous monotone function) if the solution is defined for all $t \geq 0$ and

$$
\lim _{t \rightarrow+\infty} \psi(t)=\pi^{-} ; \varlimsup_{t \rightarrow+\infty} \psi(t)=\pi^{+} ;-\infty<\pi^{-}<\pi^{+}<+\infty .
$$

The solution $\mathbf{x}\left(t, \mathbf{x}_{0}\right)$ with $\mathbf{x}_{0} \in R^{n}$ of the system (1) is called oscillating, if there exist some output $\psi$ and constants $\pi^{-}, \pi^{+}$such that $\mathbf{x}\left(t, \mathbf{x}_{0}\right)$ is $\left[\pi^{-}, \pi^{+}\right]$-oscillation with respect to the output $\psi$. The forward complete system (1) is called oscillatory, if for almost all $\mathbf{x}_{0} \in R^{n}$ the solutions $\mathbf{x}\left(t, \mathbf{x}_{0}\right)$ of the system are oscillating. The oscillatory system (1) is called uniformly oscillatory, if for almost all $\mathbf{x}_{0} \in R^{n}$ for corresponding solutions $\mathbf{x}\left(t, \mathbf{x}_{0}\right)$ there exist output $\psi$ and constants $\pi^{-}, \pi^{+}$not depending on initial conditions. $\square$ In other words the solution $\mathbf{x}\left(t, \mathbf{x}_{0}\right)$ is oscillating if the output $\psi(t)=\eta\left(\mathbf{x}\left(t, \mathbf{x}_{0}\right)\right)$ is asymptotically bounded and there is no single limit value of $\psi(t)$ for $t \rightarrow+\infty$. Note that the term "almost all solutions" is used to emphasize that generally the system (1) has a nonempty set of equilibrium points, thus, there exists a set of initial conditions with zero measure such that the corresponding solutions are not oscillating.

The o r e m 4 (Efimov and Fradkov, 2009). Let the system (1) have two locally Lipschitz continuous Lyapunov functions $V_{1}$ and $V_{2}$ possessing the following inequalities for all $\mathbf{x} \in R^{n}\left(v_{1}, v_{2}, v_{3}, v_{4} \in K_{\infty}\right):$

$$
v_{1}(|\mathbf{x}|) \leq V_{1}(\mathbf{x}) \leq v_{2}(|\mathbf{x}|), v_{3}(|\mathbf{x}|) \leq V_{2}(\mathbf{x}) \leq v_{4}(|\mathbf{x}|),
$$

and for some $0<X_{1}<v_{1}^{-1} \circ v_{2} \circ v_{3}^{-1} \circ v_{4}\left(X_{2}\right)<+\infty$ :

$$
\begin{gathered}
D V_{1}(\mathbf{x}) \mathbf{f}(\mathbf{x})>0 \text { for all } 0<|\mathbf{x}|<X_{1} \text { and } \mathbf{x} \notin \Xi ; \\
D V_{2}(\mathbf{x}) \mathbf{f}(\mathbf{x})<0 \text { for all }|\mathbf{x}|>X_{2} \text { and } \mathbf{x} \notin \Xi,
\end{gathered}
$$

where $\Xi \subset R^{n}$ is a set with zero Lebesgue measure contained all equilibriums of the system, and

$$
\Omega \cap \Xi=\varnothing, \Omega=\left\{\mathbf{x}: v_{2}^{-1} \circ v_{1}\left(X_{1}\right)<|\mathbf{x}|<v_{3}^{-1} \circ v_{4}\left(X_{2}\right)\right\} .
$$

Then the system (1) is oscillatory.
The Lyapunov function for the linearised system (1) at the origin is a candidate for the function $V_{1}$ (Yakubovich, 1975). Instead of existence of the function $V_{2}$ one can require just boundedness of the system (1) solutions with known upper bound (this fact can be verified using another approach not dealing with Lyapunov functions analysis).

The or em 5 (Efimov and Fradkov, 2009). Let the system (1) be uniformly oscillatory with respect to the output $\psi=\eta(\mathbf{x})$ (where $\eta: R^{n} \rightarrow R$ is a continuous monotone function), and for all $\mathbf{x} \in R^{n}$ the following relations are satisfied:

$$
\chi_{1}(|\mathbf{x}|) \leq \eta(\mathbf{x}) \leq \chi_{2}(|\mathbf{x}|), \chi_{1}, \chi_{2} \in K_{\infty} ;
$$

the set of initial conditions for which the system is not oscillating consists in just one point $\Xi=\{\mathbf{x}: \mathbf{x}=0\}$. Then there exist two locally Lipschitz continuous Lyapunov functions $V_{1}: R^{n} \rightarrow R_{+}$and $V_{2}: R^{n} \rightarrow R_{+}$such that for all $\mathbf{x} \in R^{n}$ the inequalities hold $\left(v_{1}, v_{2}, v_{3}, v_{4} \in K_{\infty}\right)$ :

$$
\begin{gathered}
v_{1}(|\mathbf{x}|) \leq V_{1}(\mathbf{x}) \leq v_{2}(|\mathbf{x}|), v_{3}(|\mathbf{x}|) \leq V_{2}(\mathbf{x}) \leq v_{4}(|\mathbf{x}|) \\
D V_{1}(\mathbf{x}) \mathbf{f}(\mathbf{x})>0 \text { for all } 0<|\mathbf{x}|<\chi_{2}^{-1}\left(\pi^{-}\right) \\
D V_{2}(\mathbf{x}) \mathbf{f}(\mathbf{x})<0 \text { for all }|\mathbf{x}|>\chi_{1}^{-1}\left(\pi^{+}\right)
\end{gathered}
$$

The theorems 4 and 5 present the sufficient and necessary conditions for the system (1) to be oscillatory. Being rather simple these conditions can be useful in different applications (Efimov and Fradkov, 2008; Efimov and Fradkov, 2009). Unfortunately, in some situations these conditions could be restrictive. For example, in sufficient part they need the knowledge of two Lyapunov functions for the system (1), that can be an ambiguous requirement. Additionally, the conditions are oriented to the locally unstable origin case, however, a system with several limit cycles can have a locally stable origin. These shortages can be resolved applying homogeneity approach as it is shown below.

\section{MAIN RESULTS}

For the homogeneous systems the functions $V_{1}$ and $V_{2}$ can be chosen according to the corresponding approximations at the origin or at infinity. For this purpose we are going to develop the Lyapunov theorems for locally homogeneous unstable/stable systems and apply them to detect the oscillations presence next.

\subsection{Unstable homogeneous systems}

At the first we present an analogue of Theorem 1 for local instability of the homogeneous system (1) at the origin.

Lemma 1. For the system (1) with $\mathbf{r}$-homogeneous and continuous function $\mathbf{f}: R^{n} \rightarrow R^{n}$ the following properties are equivalent:

- the system (1) is (locally) strongly unstable, i.e. there exists $\delta>0$ such that for any $0<\left|\mathbf{x}_{0}\right|<\delta$ there exists $T_{\mathbf{x}_{0}}$ such that $\left|\mathbf{x}\left(t, \mathbf{x}_{0}\right)\right|>\delta$ for all $t \geq T_{\mathbf{x}_{0}}$;

- there exists continuously differentiable homogeneous 
Lyapunov function $V: R^{n} \rightarrow R_{+}$such that for all $\mathbf{x} \in R^{n}$,

$$
\begin{gathered}
\alpha_{1}(|\mathbf{x}|) \leq V(\mathbf{x}) \leq \alpha_{2}(|\mathbf{x}|), D V(\mathbf{x}) \mathbf{f}(\mathbf{x}) \geq \alpha(|\mathbf{x}|) \\
V\left(\Lambda_{\mathbf{r}} \mathbf{x}\right)=\lambda^{k} V(\mathbf{x}), k \geq 0
\end{gathered}
$$

for some $\alpha_{1}, \alpha_{2} \in K_{\infty}, \alpha \in K$.

All proofs are excluded due to space limitations.

3.2. Stability/instability conditions for locally homogeneous systems

An advantage of the $\mathbf{r}$-homogeneous system (1) is that the global behavior of the system can be completely characterized by the behavior of the system on the sphere with the unit radius $S_{\mathbf{r}}$. To explain this property let us introduce the coordinate transformation $\mathbf{x}=\boldsymbol{\Lambda}_{\mathbf{r}} \mathbf{y}$ that connects any $\mathbf{x} \in R^{n}$ with $\mathbf{y} \in S_{\mathbf{r}}$ for suitably chosen $\lambda \geq 0$. Let $V: R^{n} \rightarrow R_{+}$be a continuously differentiable homogeneous Lyapunov function (as used in Theorem 1, Lemma 1), then

$$
\begin{aligned}
D V(\mathbf{x}) \mathbf{f}(\mathbf{x}) & =D V\left(\boldsymbol{\Lambda}_{\mathbf{r}} \mathbf{y}\right) \mathbf{f}\left(\boldsymbol{\Lambda}_{\mathbf{r}} \mathbf{y}\right)= \\
& =\lambda^{d+k} D V(\mathbf{y}) \mathbf{f}(\mathbf{y})=|\mathbf{x}|_{\mathbf{r}}^{d+k} D V(\mathbf{y}) \mathbf{f}(\mathbf{y}),
\end{aligned}
$$

where $d$ is the homogeneity degree of the function $\mathbf{f}$ and $k$ is the degree of the Lyapunov function $V$. Therefore, sign definiteness of the function $V$ derivative can be checked on the sphere $S_{\mathbf{r}}$ only.

For the $\left(\mathbf{r}, \lambda_{0}, \mathbf{f}_{0}\right)$-homogeneous system (1) this technique establishes the relation between the global stability properties of the approximating dynamics

$$
\dot{\mathbf{x}}=\mathbf{f}_{0}(\mathbf{x})
$$

and the local ones of the original system (1).

L e m m a 2 . Let the system (1) be $\left(\mathbf{r}, \lambda_{0}, \mathbf{f}_{0}\right)$-homogeneous, the functions $\mathbf{f}: R^{n} \rightarrow R^{n}$ and $\mathbf{f}_{0}: R^{n} \rightarrow R^{n}$ be continuous and the approximating dynamics (2) have $\mathbf{r}$-homogeneous and continuously differentiable Lyapunov function $V_{0}: R^{n} \rightarrow R_{+}, \quad \alpha_{1}(|\mathbf{x}|) \leq V_{0}(\mathbf{x}) \leq \alpha_{2}(|\mathbf{x}|), \alpha_{1}, \alpha_{2} \in K_{\infty}$ for all $\mathbf{x} \in R^{n}$.

(i) Let $a=-\sup _{\mathbf{y} \in S_{\mathbf{r}}} D V_{0}(\mathbf{y}) \mathbf{f}_{0}(\mathbf{y}), a>0$, then

1) if $\lambda_{0}=0$, then there exists $0<\bar{\lambda}_{\varepsilon}$ such that the system (1) is locally asymptotically stable with the domain of asymptotic stability containing the set

$$
X_{0}=\left\{\mathbf{x} \in R^{n}:|\mathbf{x}| \leq \alpha_{1}^{-1} \circ \alpha_{2} \circ \underline{\sigma}_{\mathbf{r}}\left(\bar{\lambda}_{\varepsilon}\right)\right\} ;
$$

2) if $\lambda_{0}=+\infty$, then there exists $0<\underline{\lambda}_{\varepsilon}<+\infty$ such that (1) is globally asymptotically stable with respect to the forward invariant set $X_{\infty}=\left\{\mathbf{x} \in R^{n}:|\mathbf{x}| \leq \alpha_{1}^{-1} \circ \alpha_{2} \circ \bar{\sigma}_{\mathbf{r}}\left(\underline{\lambda}_{\varepsilon}\right)\right\}$;

$3)$ if $0<\lambda_{0}<+\infty$, then there exist $0<\underline{\lambda}_{\varepsilon} \leq \lambda_{0} \leq \bar{\lambda}_{\varepsilon}<+\infty$ such that the system (1) is finite time asymptotically stable with respect to the forward invariant set $X_{\infty}$ with the region of attraction

$$
X=\left\{\mathbf{x} \in R^{n}: \alpha_{1}^{-1} \circ \alpha_{2} \circ \bar{\sigma}_{\mathbf{r}}\left(\underline{\lambda}_{\varepsilon}\right)<|\mathbf{x}|<\alpha_{1}^{-1} \circ \alpha_{2} \circ \underline{\sigma}_{\mathbf{r}}\left(\bar{\lambda}_{\varepsilon}\right)\right\}
$$

provided that the set $X$ is connected and $X \neq \varnothing$.

(ii) Let $a=\inf _{\mathbf{y} \in S_{\mathbf{r}}} D V_{0}(\mathbf{y}) \mathbf{f}_{0}(\mathbf{y}), a>0$, then

1) if $\lambda_{0}=0$, then there exists $0<\bar{\lambda}_{\varepsilon}$ such that the system (1) is asymptotically stable with respect to the forward invariant set $R^{n} \backslash X_{0}$ with the region of attraction $X_{0} \backslash\{0\}$;

2) if $\lambda_{0}=+\infty$, then there exists $0<\underline{\lambda}_{\varepsilon}<+\infty$ such that the set $R^{n} \backslash X_{\infty}$ is forward invariant for the system (1);

3) if $0<\lambda_{0}<+\infty$, then there exist $0<\underline{\lambda}_{\varepsilon} \leq \lambda_{0} \leq \bar{\lambda}_{\varepsilon}<+\infty$ such that the system (1) is finite time asymptotically stable with respect to the forward invariant set $R^{n} \backslash X_{0}$ with the region of attraction $X$ provided that the set $X$ is connected and $X \neq \varnothing$.

In other words the result of Lemma 2 means that the behavior of the system (1) is inherited after (2) into the set $X_{\mathbf{r}}=\left\{\mathbf{x} \in R^{n}: \underline{\lambda}_{\varepsilon}<|\mathbf{x}|_{\mathbf{r}}<\bar{\lambda}_{\varepsilon}\right\}$ provided that it contains an equilevel set of the function $V_{0}$ (the set $X$ is connected and not empty). The first two parts of the case (i) correspond to theorems 2 and 3 . If we assume that the approximating vector field $\mathbf{f}_{0}$ is $\mathbf{r}$-homogeneous, then the requirement on existence of the $\mathbf{r}$-homogeneous Lyapunov function $V_{0}$ follows by Theorem 1 and Lemma 1 results. The conditions of the lemma can be relaxed skipping homogeneity and $\mathbf{f}, \mathbf{f}_{0}$ continuity assumptions as follows.

Corollary 1 . Let $\mathbf{r}>0, \lambda_{0} \geq 0$ and $\mathbf{f}_{0}: R^{n} \rightarrow R^{n}$ be given and the approximating dynamics (2) have $\mathbf{r}$ homogeneous and continuously differentiable Lyapunov function $V_{0}: R^{n} \rightarrow R_{+}, \alpha_{1}(|\mathbf{x}|) \leq V_{0}(\mathbf{x}) \leq \alpha_{2}(|\mathbf{x}|), \alpha_{1}, \alpha_{2} \in K_{\infty}$ for all $\mathbf{x} \in R^{n}$. Let one of the following properties hold

(i) $a=-\sup _{\mathbf{y} \in S_{\mathbf{r}}} D V_{0}(\mathbf{y}) \mathbf{f}_{0}(\mathbf{y}), a>0$;

(ii) $a=\inf _{\mathbf{y} \in S_{\mathbf{r}}} D V_{0}(\mathbf{y}) \mathbf{f}_{0}(\mathbf{y}), a>0$,

and there exist $\underline{\lambda}_{\varepsilon} \leq \lambda_{0} \leq \bar{\lambda}_{\varepsilon}$ such that

$$
\sup _{\mathbf{y} \in S_{\mathbf{r}}}\left|D V_{0}(\mathbf{y})\left[\lambda^{-d} \boldsymbol{\Lambda}_{\mathbf{r}}^{-1} \mathbf{f}\left(\boldsymbol{\Lambda}_{\mathbf{r}} \mathbf{y}\right)-\mathbf{f}_{0}(\mathbf{y})\right]\right|<a
$$

for all $\lambda \in\left(\underline{\lambda}_{\varepsilon}, \bar{\lambda}_{\varepsilon}\right)$, then all claims (i),1-(i),3 and (ii),1-(ii),3 of Lemma 2 are valid.

Let us apply these results for oscillations detection.

\subsection{Oscillations in locally homogeneous systems}

Let $0 \leq \lambda_{0} \leq \ldots \leq \lambda_{N} \leq+\infty$ be an ordered sequence for a given integer $N>0$.

Theorem 6. Let the system (1) be $\left(\mathbf{r}_{j}, \lambda_{j}, \mathbf{f}_{j}\right)$ homogeneous for $j=\overline{1, N}$, the functions $\mathbf{f}: R^{n} \rightarrow R^{n}$ and $\mathbf{f}_{j}: R^{n} \rightarrow R^{n}, j=\overline{1, N}$ be continuous and the locally approximating dynamical systems $\dot{\mathbf{x}}=\mathbf{f}_{j}(\mathbf{x}), j=\overline{1, N}$ have $\mathbf{r}_{j}$-homogeneous and continuously differentiable Lyapunov functions $\quad V_{j}: R^{n} \rightarrow R_{+}, \quad \alpha_{1, j}(|\mathbf{x}|) \leq V_{j}(\mathbf{x}) \leq \alpha_{2, j}(|\mathbf{x}|)$, 
$\alpha_{1, j}, \alpha_{2, j} \in K_{\infty}$ for all $\mathbf{x} \in R^{n}$ and $j=\overline{1, N}$. Let $\Xi \subset R^{n}$ be the set containing all equilibriums of the system (1).

Let one of the following conditions hold.

(i) There exists $1 \leq j^{*}<N$ such that

$$
\begin{gathered}
a_{j^{*}}=\inf _{\mathbf{y} \in S_{\mathbf{r}_{j^{*}}}} D V_{j^{*}}(\mathbf{y}) \mathbf{f}_{j^{*}}(\mathbf{y})>0 \\
a_{j^{*}+1}=-\sup _{\mathbf{y} \in S_{\mathbf{r}_{j^{*}+1}} D V_{j^{*}+1}(\mathbf{y}) \mathbf{f}_{j^{*}+1}(\mathbf{y})>0}
\end{gathered}
$$

and the sets

$$
X_{k}=\left\{\mathbf{x} \in R^{n}: \alpha_{1, k}^{-1} \circ \alpha_{2, k} \circ \bar{\sigma}_{\mathbf{r}_{k}}\left(\underline{\lambda}_{k}\right)<|\mathbf{x}|<\alpha_{1, k}^{-1} \circ \alpha_{2, k} \circ \underline{\sigma}_{\mathbf{r}_{k}}\left(\bar{\lambda}_{k}\right)\right\}
$$

for $k=j^{*}, j^{*}+1$ are connected and non empty where

$$
\sup _{\mathbf{y} \in S_{\mathbf{r}_{k}}}\left|D V_{k}(\mathbf{y})\left[\lambda^{-d} \Lambda_{\mathbf{r}_{k}}^{-1} \mathbf{f}\left(\boldsymbol{\Lambda}_{\mathbf{r}_{k}} \mathbf{y}\right)-\mathbf{f}_{k}(\mathbf{y})\right]\right|<a_{k}
$$

for all $\lambda \in\left(\underline{\lambda}_{k}, \bar{\lambda}_{k}\right), \underline{\lambda}_{k} \leq \lambda_{k} \leq \bar{\lambda}_{k}$ (such constants $\underline{\lambda}_{k}, \bar{\lambda}_{k}$ exist due to homogeneity assumption), $k=j^{*}, j^{*}+1$, and

$$
\begin{gathered}
\Omega \cap \Xi=\varnothing, \Omega=\Omega_{j^{*}} \cap \Omega_{j^{*}+1}, \\
\Omega_{j^{*}}=R^{n} \backslash\left\{\mathbf{x} \in R^{n}:|\mathbf{x}|<\alpha_{1, j^{*}}^{-1} \circ \alpha_{2, j^{*} \circ} \underline{\sigma}_{j^{*}}\left(\bar{\lambda}_{j^{*}}\right)\right\}, \\
\Omega_{j^{*}+1}=\left\{\mathbf{x} \in R^{n}:|\mathbf{x}|<\alpha_{1, j^{*}+1}^{-1} \circ \alpha_{2, j^{*}+1} \circ \bar{\sigma}_{\mathbf{r}_{j^{*}+1}}\left(\underline{\lambda}_{j^{*+1}}\right)\right\} ;
\end{gathered}
$$

(ii) There exists $1 \leq j^{*}<N$ such that

$$
\begin{gathered}
a_{j^{*}}=-\sup _{\mathbf{y} \in S_{\mathbf{r}_{j}}} D V_{j^{*}}(\mathbf{y}) \mathbf{f}_{j^{*}}(\mathbf{y})>0, \\
a_{j^{*+1}}=\inf _{\mathbf{y} \in S_{\mathbf{r}_{j^{*}+1}} D V_{j^{*}+1}(\mathbf{y}) \mathbf{f}_{j^{*}+1}(\mathbf{y})>0}
\end{gathered}
$$

and the sets $X_{k}, k=j^{*}, j^{*}+1$ are connected and non empty where $\underline{\lambda}_{k} \leq \lambda_{k} \leq \bar{\lambda}_{k}$ are defined as for the case (i), and

$\Omega \cap \Xi=\varnothing$

$$
\begin{gathered}
\Omega=\left\{\mathbf{x} \in R^{n}: \alpha_{1, j^{*}}^{-1} \circ \alpha_{2, j^{*}} \circ \underline{\sigma}_{\mathbf{r}_{j^{*}}}\left(\bar{\lambda}_{j^{*}}\right) \leq|\mathbf{x}| \leq\right. \\
\left.\leq \alpha_{1, j^{*}+1}^{-1} \circ \alpha_{2, j^{*}+1} \circ \bar{\sigma}_{\mathbf{r}_{j^{*}+1}}\left(\underline{\lambda}_{j^{*}+1}\right)\right\} .
\end{gathered}
$$

Then (1) has oscillating trajectories into the set $\Omega$.

The result of the last theorem says that if the system (1) is locally homogeneous and unstable in an inner (outer) subset and locally homogeneous stable in an outer (inner) subset, then between these subsets should exist an invariant set containing oscillating trajectory providing that the equilibriums are excluded from this region. The conditions of Theorem 6 can be relaxed taking in mind the result of Corollary 1 (this reformulation is omitted here for brevity of presentation). The set $\Omega$ can be used to estimate the constants $\pi^{-}, \pi^{+}$, i.e. to estimate the amplitude of oscillation.

\section{EXAMPLES}

Consider the system

$$
\begin{gathered}
\dot{x}_{1}=-x_{1}+x_{2}+2 \tanh \left(x_{1}\right) ; \dot{x}_{2}=-2 x_{1}+x_{3} ; \\
\dot{x}_{3}=-1.5 x_{1}+2 \tanh \left(x_{1}\right),
\end{gathered}
$$

where $\mathbf{x}=\left[\begin{array}{lll}x_{1} & x_{2} & x_{3}\end{array}\right]^{T} \in R^{3}$ is the state vector, and the system (3) is in Lurie form (linear asymptotically stable system closed by nonlinear feedback). The system (3) has the single equilibrium at the origin, it is homogeneous in the bi-limit, namely $\left(\mathbf{r}_{j}, \lambda_{j}, \mathbf{f}_{j}\right)$-homogeneous with $j=1,2$ and

$$
\begin{gathered}
\mathbf{r}_{1}=\left[\begin{array}{lll}
0.5 & 0.5 & 0.5
\end{array}\right], \lambda_{1}=0, \mathbf{f}_{1}(\mathbf{x})=\mathbf{A}_{1} \mathbf{x} ; \\
\mathbf{r}_{2}=\left[\begin{array}{lll}
0.5 & 0.5 & 0.5
\end{array}\right], \lambda_{2}=+\infty, \mathbf{f}_{2}(\mathbf{x})=\mathbf{A}_{2} \mathbf{x} ; \\
\mathbf{A}_{1}=\left[\begin{array}{ccc}
1 & 1 & 0 \\
-2 & 0 & 1 \\
0.5 & 0 & 0
\end{array}\right], \mathbf{A}_{2}=\left[\begin{array}{ccc}
-1 & 1 & 0 \\
-2 & 0 & 1 \\
-1.5 & 0 & 0
\end{array}\right]
\end{gathered}
$$

with zero degree. All eigenvalues of the matrix $\mathbf{A}_{1}$ have positive real parts, and all eigenvalues of the matrix $\mathbf{A}_{2}$ have negative real parts. Therefore, the conditions of Theorem 6 hold and the system (3) is oscillating, actually the results of its simulation presented in Fig. 1 show, that it has the stable limit cycle.

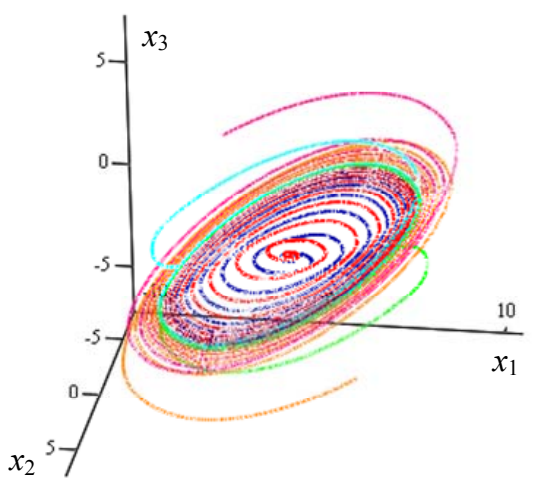

Fig. 1. The results of the system (3) simulation.

Next, consider the system

$$
\begin{aligned}
& \dot{x}_{1}=2 x_{1}-\left[0.5 \pi-x_{1}^{2}-x_{2}^{2}\right]^{2} x_{1}^{3}-3\left(x_{1}-x_{2}\right) \cos \left(x_{1}^{2}+x_{2}^{2}\right) ;(4) \\
& \dot{x}_{2}=x_{2}-\left[0.4 \pi-x_{1}^{2}-x_{2}^{2}\right]^{2} x_{2}^{3}-2\left(2 x_{2}-x_{1}\right) \cos \left(x_{1}^{2}+x_{2}^{2}\right),
\end{aligned}
$$

where $\mathbf{x}=\left[\begin{array}{ll}x_{1} & x_{2}\end{array}\right]^{T} \in R^{2}$. The origin is the only equilibrium of the system. This system is homogeneous in three limits:

$$
\begin{gathered}
\mathbf{r}_{1}=\left[\begin{array}{ll}
0.5 & 0.5
\end{array}\right], \lambda_{1}=0 ; \mathbf{r}_{2}=\left[\begin{array}{ll}
0.5 & 0.5
\end{array}\right], \lambda_{2}=+\infty ; \\
\mathbf{r}_{3}=\left[\begin{array}{ll}
0.5 & 0.5
\end{array}\right], \lambda_{3}=0.5 \pi ; \\
\mathbf{f}_{1}(\mathbf{x})=\left[\begin{array}{c}
-x_{1}+3 x_{2} \\
-3 x_{2}-2 x_{1}
\end{array}\right], \mathbf{f}_{2}(\mathbf{x})=-\left(x_{1}^{2}+x_{2}^{2}\right)^{2}\left[\begin{array}{l}
x_{1}^{3} \\
x_{2}^{3}
\end{array}\right], \\
\mathbf{f}_{3}(\mathbf{x})=\left[\begin{array}{c}
2 x_{1} \\
\left\{1-0.005 \pi^{3}\left[x_{2}^{2} /\left(x_{1}^{2}+x_{2}^{2}\right)\right]\right\} x_{2}
\end{array}\right]
\end{gathered}
$$

with $d=0$ for all cases. In all three modes the approximating dynamics have Lyapunov function $V(\mathbf{x})=0.5\left(x_{1}^{2}+x_{2}^{2}\right)$, and it is easy to verify that the vector fields $\mathbf{f}_{1}, \mathbf{f}_{2}$ are asymptotically stable and $\mathbf{f}_{3}$ is unstable. Thus, the conditions of Theorem 6 can be verified twice signalizing that the system has two limit cycles (the system (4) is planar, thus any isolated oscillating trajectory is a limit cycle). The results of simulation presented in Fig. 2 confirm this conclusion. 


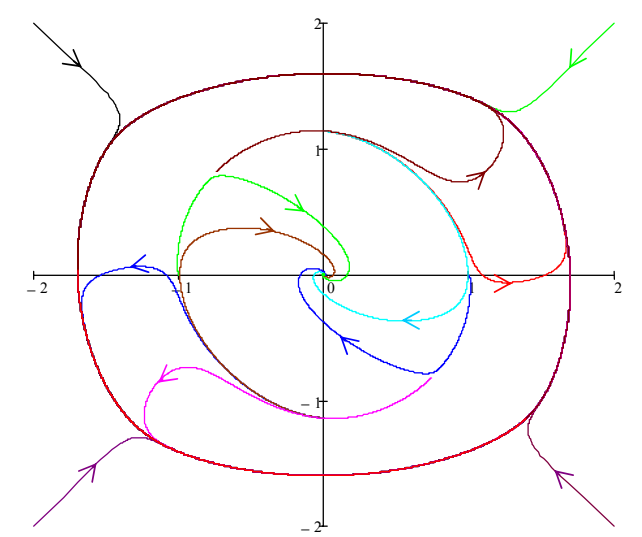

Fig. 2. The results of the system (4) simulation.

\section{CONCLUSION}

The paper presents conditions of oscillations existence for locally homogeneous systems. The conditions allows us to detect multiple limit cycles presence for homogeneous vector fields in several limits. Efficiency of the proposed conditions demonstrated on several examples. Partly the proposed conditions interact with the second part of Hilbert's 16th problem that addresses the issue of evaluation of an upper bound for the number of limit cycles in polynomial vector fields of degree $\mathrm{n}$ and investigation their relative positions (for the planar system (1)). Homogeneity is naturally satisfied for the polynomial vector fields, verifying local homogeneity conditions proposed in this work and checking conditions of Theorem 6 it is possible to estimate the maximum number of limit cycles and localize their positions. That is rest for future works.

\section{REFERENCES}

Andrieu V., Praly L., Astolfi A. (2008). Homogeneous Approximation, Recursive Observer Design, and Output Feedback. SIAM J. Control Optimization, 47(4), pp. 1814-1850.

Bacciotti A., Rosier L. (2001). Liapunov Functions and Stability in Control Theory. Lecture Notes in Control and Inform. Sci., 267, Springer, Berlin.

Bhat S.P., Bernstein D.S. (2005). Geometric homogeneity with applications to finite-time stability. Mathematics of Control, Signals and Systems, 17, pp. 101-127.

Efimov D.V., Fradkov A.L. (2009). Oscillatority of Nonlinear Systems with Static Feedback. SIAM J. Control Optimization, 48(2), pp. 618-640.

Efimov D.V., Fradkov A.L. (2008). Yakubovich's Oscillatority of Circadian Oscillations Models. Mathematical Biosciences, 216, pp. 187-191.

Fradkov A.L., Pogromsky A.Yu. (1998). Introduction to oscillations and chaos. World Scientific, Singapore.

Grüne L. (2000). Homogeneous state feedback stabilization of homogeneous systems. SIAM J. Control Optimization, 38(4), pp. 1288-1314.

Hermes H. (1991a). Nilpotent and high-order approximations of vector field systems. SIAM Review, 33(2), pp. $238-264$.

Hermes H. (1991b). Homogeneous coordinates and continuous asymptotically stabilizing feedback controls. In Dif- ferential Equations: Stability and Control, Lecture Notes in Pure Appl. Math.,109, S. Elaydi, ed., Marcel Dekker,pp.249-260.

Hong Y. (2002). Finite-time stabilization and stabilizability of a class of controllable systems. Systems\&Control Lett., 46, pp. 231-236.

Kawski M. (1991). Homogeneous feedback stabilization. In Progress in systems and control theory: Vol.7. New trends in systems theory. Birkhuser.

Khalil H.K. (2002). Nonlinear Systems. 3rd edition, Prentice Hall, Upper Saddle River, New Jersey.

Leonov G.A., Burkin I.M., Shepelyavyi A.I. (1995). Frequency Methods in Oscillation Theory. Kluwer, Dordrecht. (in Russian: 1992)

Lin Y., Sontag E.D., Wang Y. (1996). A Smooth Converse Lyapunov Theorem for Robust Stability. SIAM J. Control Optimization, 34, pp. 124-160.

Martinez S., Cortes J., Bullo F. (2003). Analysis and design of oscillatory control systems. IEEE Trans. Aut. Contr., 48, 7, pp. 1164-1177.

Di Meglio F., Kaasa G.-O., Petit N. (2009). A first principle model for multiphase slugging flow in vertical risers. Proc. $48^{\text {th }}$ IEEE Conf. on Decision and Control 2009, pp. 8244-8251.

Moulay E., Perruquetti W. (2006). Finite time stability and stabilization of a class of continuous systems. J. Mathematical Analysis Applications, 323(2), pp. 1430-1443.

Rosier L. (1992). Homogeneous Lyapunov function for homogeneous continuous vector field. Systems\&Control Lett., 19, pp. 467-473.

Rothschild L.P., Stein E.M. (1976). Hypoelliptic differential operators and nilpotent groups. Acta Mathematica, 137, pp. 247-320.

Sepulchre R., Aeyels D. (1996). Stabilizability does not imply homogeneous stabilizability for controllable systems. SIAM J. Control Optimization, 34(5), pp. 1798-1813.

Sinègre L., Petit N., Lemétayer P., Gervaud P., Ménégatti P. (2005). Casing-heading phenomenon in gas-lifted well as a limit cycle of a 2D model with switches. Proc. 2005 IFAC World Congress.

Yakubovich V.A. (1973). Frequency oscillations conditions in nonlinear systems with stationary single nonlinearity. Siberian math journal, 14, № 2.

Yakubovich V.A. (1975). Oscillations in systems with discontinuous and hysteresis nonlinearities. Automation and Remote Control, 12.

Yakubovich V.A., Tomberg E.A. (1989). Conditions for selfinduced oscillations in nonlinear systems, Siberian Math. J., 30, pp. 641-653. 